(RESEARCh ARTICLE)

\title{
SVM Kernels comparison for brain tumor diagnosis using MRI
}

\author{
Wedad Abdul Khuder Naser *, Eman Abdulmunem Kadim and Safana Hyder Abbas \\ Department of computer science, University Al Mustansiriyah, Baghdad, Iraq.
}

Global Journal of Engineering and Technology Advances, 2021, 07(02), 026-036

Publication history: Received on 30 March 2021; revised on 02 May 2021; accepted on 05 May 2021

Article DOI: https://doi.org/10.30574/gjeta.2021.7.2.0065

\begin{abstract}
Magnetic Resonance Image (MRI) brain images have an essential role in medical analysis and cancer identification .In this paper multi kernel SVM algorithm is used for MRI brain tumor detection. The proposed work is involving the following stages: image acquisition, image preprocessing, feature extraction and tumor classification. An automatic threshold selection region based segmentation method called Otsu is used for thresholding during preprocessing stage. SVM classification algorithm with four different kernels are used to determine the normal and abnormal images. SVM with quadratic kernel results in best classification accuracy of $86.5 \%$.
\end{abstract}

Keywords: Brain tumors; Support vector machine; Kernels; Image processing; Magnetic Resonance Image (MRI)

\section{Introduction}

Because of the unusual brain growth of cells, humans are severely affected by brain tumors. It can affect the proper function of the brain and can be a serious threat to life. There are two kinds of brain tumors, benign and malignant. Benign tumors are less risky than malignant tumors as malignant ones develop quickly and dangerously while benign tumors grow slowly and harmlessly. Medical imagery method is used to provide an internal human body visual illustration for health purposes and this technique can detect noninvasive possibilities. Based on noninvasive method, different kinds of medical imaging technologies like; MRI, Ultrasound, X-ray, PET, SPECT and CT scan are used [1]. Magnetic resonance imaging (MRI) is used in the majority and offers a greater contrast in the brain with cancer tissue compared with other medical imaging methods. For brain tumor identification, MRI images may therefore be used. In this paper an MRI brain tumor is recognized using SVM algorithm with four different kernels.

This paper is organized as follows: Section 2 provides a review of related works; then a brief explanation of SVM algorithm is described in section3. Section 4 shows the detailed steps of the used methodology. Section 5 presents the experimental result and finally section 6 summarizes the conclusions and finally the list of references.

\section{Related works}

Many studies on MRI brain image segmentation and tumor detection techniques have been proposed. The following is a short review of some of these studies:

A hybrid segmentation technique is proposed for detection of MRI image brain tumor. Author combined FCM and seed region growing algorithm as hybrid technique for segmentation. This method outputted average segmentation score $\mathrm{S}$ of $90 \%$ with noise level of $3 \%$ and $9 \%$, respectively [2].

\footnotetext{
${ }^{*}$ Corresponding author: Wedad Abdul Khuder

Department of computer science, University Al Mustansiriyah Baghdad, Iraq.

Copyright $(2021$ Author(s) retain the copyright of this article. This article is published under the terms of the Creative Commons Attribution Liscense 4.0.
} 
A neural network technique for MR image brain tumor detection and classification has been presented. This method has an accuracy of $83 \%$ and segmented white matter, Gray matter, CSF and obtained quality rate separately [3].

Developed a localized fuzzy clustering method to segment MRI images. The author used jaccard similarity index as a metric to obtain accuracy. This localized fuzzy clustering method obtained the segmentation accuracy of 95\% [4].

A novel tissue segmentation algorithm by combination of wavelets and neural networks, providing effective segmentation of brain MRI images into a tumor, was proposed. Features based on textures are then extracted using the segmentation and classification tool of the artificial neural network (ANN)[5].

\section{SVM (Support Vector Machine)}

SVM is an algorithm of supervised learning based on the statistical learning concept. Just let a labeled data set (training set): $D=\{|x, y| \mid x \rightarrow$ data sample, $y \rightarrow$ class label $\}$, SVM tries to calculate the mapping function $f$ such that $f(x)=y$ for all the samples of the data set. The correlation between data samples and their specific class labels is defined in this mapping function and is used for classifying unknown different data. SVM classification is carried out using the following classification function: SVM classification.

$$
D(z)=\operatorname{sing}\left(\sum_{i=1}^{N} \propto_{i} y_{i} K\left(z, s_{i}\right)+b\right)
$$

The alpha parameters are $\propto_{i}$, the support vector's class labels are $y_{i}$, the support vectors are $S_{i}$, the input vector is z, the chosen kernel function is $K\left(Z, S_{i}\right)$, and the bias is $\mathrm{b}$. The below are four types of kernels:

Linear: $\mathrm{K}(\mathrm{X}, \mathrm{Z})=\mathrm{XOZ}$

Polynomial $: \mathrm{K}(\mathrm{X}, \mathrm{Z})=((X \bigcirc Z)+1)^{d}, d>0$

$R B F: K(X, Z)=\exp \left(-\|X-Z\|^{2} /\left(2 \sigma^{2}\right)\right)$

Quaratic: $\left\{\Phi(\mathrm{x})_{1}, \Phi(\mathrm{x})_{2}\right\}=\left\{\mathrm{x}_{1}^{2}, \mathrm{x}_{2}^{2}\right\}$
(2)

SVM adopts the idea of converting the domain of input into large dimension space to improve classification function [6].

\section{Methodology}

The proposed approach is illustrated in figure (1).

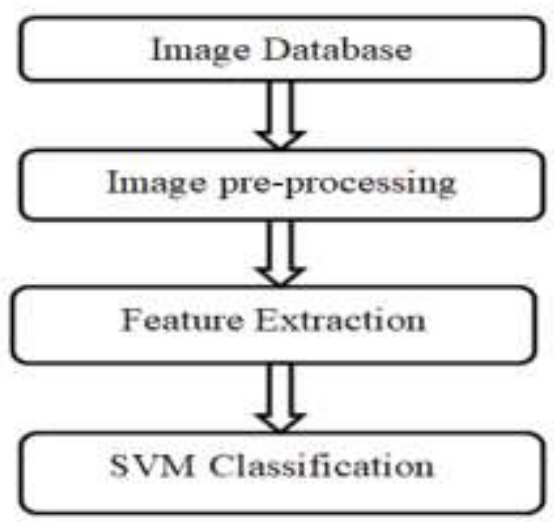

Figure 1 The proposed approach 


\subsection{Image data base}

The dataset consists of MRI brain images downloaded from Harvard Medical School's website (URL: http://med.harvard.edu/AANLIB/) and OASIS dataset (URL: http:// www.oasis-brains.org [7].

We choose 10 images for benign case and 10 images for malignant case to be applied to svm algorithm . For each case, for training 7 images are used and for testing 3 images are used.

An example of MRI images for brain tumors are shown in figure 2. a and b

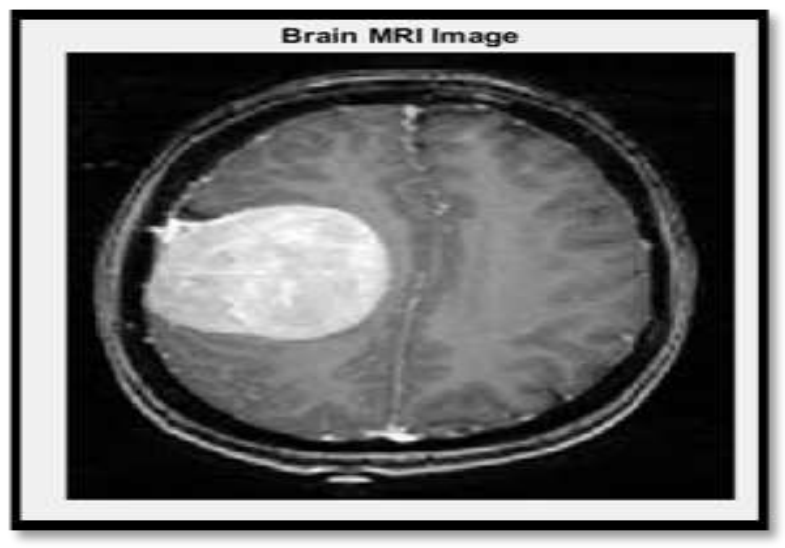

Figure 2(a) Benign Tumor

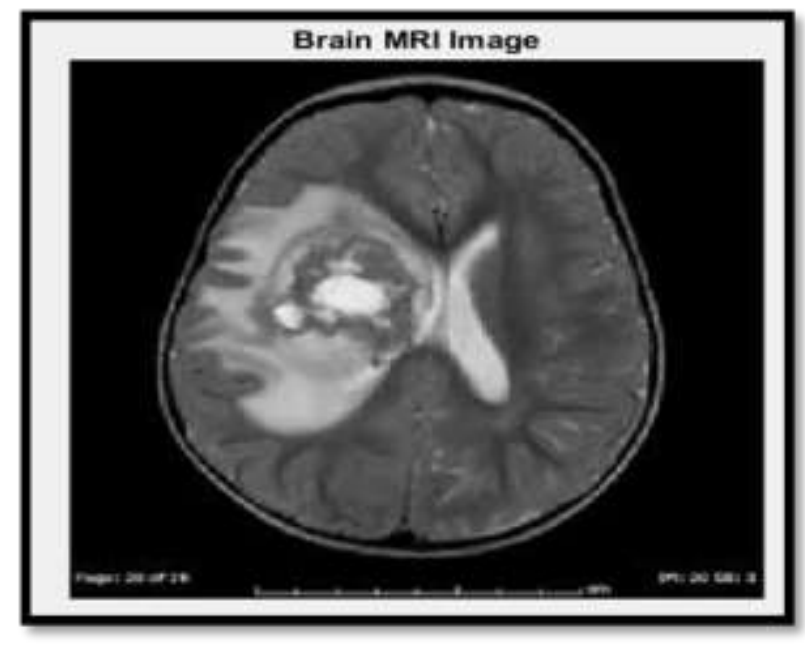

Figure 2(b) Malignant Tumor

Figure 2 Examples of brain tumors MRI images

\subsection{Image pre processing}

MRI images must be preprocessed before extracting the important features. The simplest approach to segment an image is using thresholding. Otsu, an automatic threshold selection region dependent segmentation method, is employed. The Otsu method is a type of global thresholding that it depends on the image's gray value [8,9]. By minimizing the withinclass variance of the two groups of pixels separated by the thresholding operator, Otsu's method selects the threshold. Figure (3) illustrates an Otsu thresholded image. 


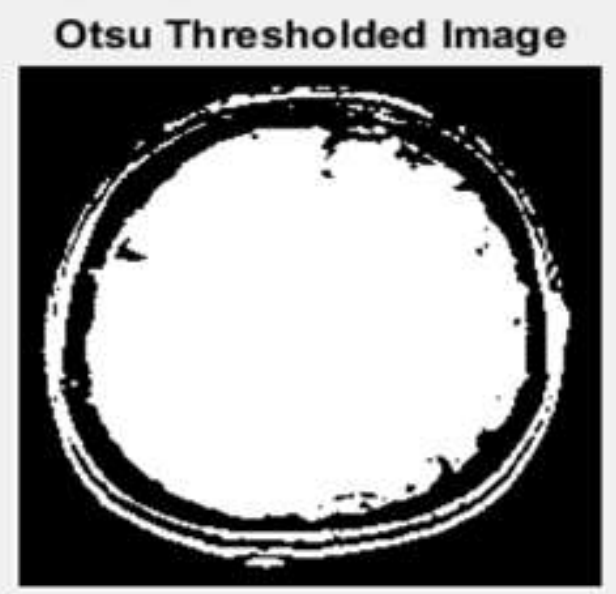

Figure 3 Otsu thresholded Image

The segmented tumor in malignant and benign cases are shown in figures (4) and (5).

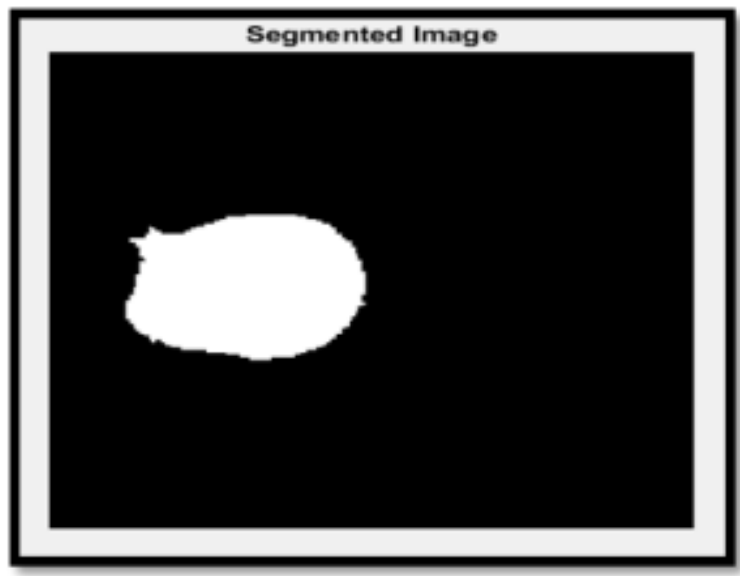

Figure 4 Segmented benign tumor image

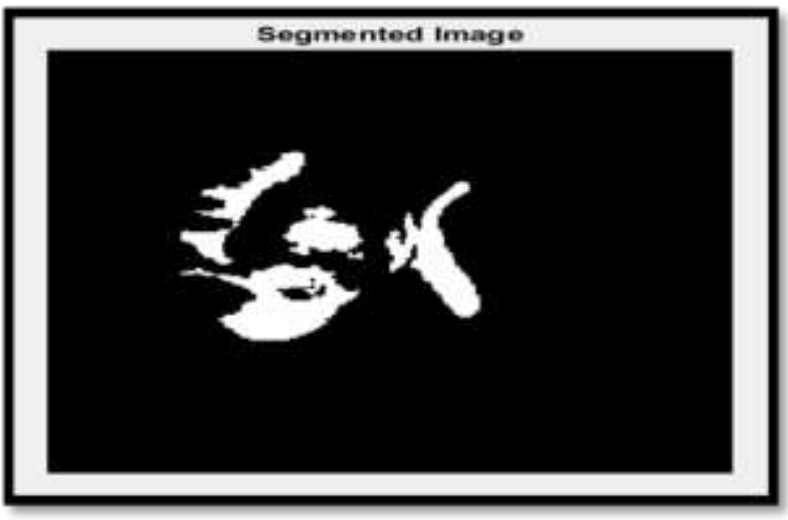

Figure 5 Segmented Malignant tumor image 


\subsection{Extracted features}

The malignant and benign MRI images of brain tumor are not visually distinguishable. Hence, different features are extracted of both benign and malignant images to determine the tumor type.

From an MRI image 13 features are extracted:

\subsubsection{The co-occurrence probabilities}

it gives a second-order method for creating texture features [10]. The probability measures can be estimated from this formula:

$$
\mathrm{Co}_{\mathrm{ij}}=\frac{\mathrm{P}_{\mathrm{ij}}}{\sum_{\mathrm{i} . \mathrm{j}=\mathbf{0}}^{\mathrm{G}} \mathbf{p}_{\mathrm{ij}}}
$$

Where:

- Coij indicates the probability of co-occurrence between grey level $\mathrm{i}$ and $\mathrm{j}$

- $\quad$ pij indicates the number of gray levels $i$ and $j$ that exist in the given window.

- $\mathrm{G}$ is the gray levels quantized number [11].

\subsubsection{Entropy Measure}

The unpredictability or complexity of the image data is measured [12]. When the image is not texturally uniform, the entropy is large. As a result, complex textures have a high entropy [11]. The following is a definition of the entropy equation.:

$$
\operatorname{Entro}=-\sum_{i, j=0}^{N g} \operatorname{Co}(i, j) \log _{2} \operatorname{Co}(i, j)
$$

Where $(\mathrm{Ng})$ is the number of gray levels

\subsubsection{Energy Measure}

It is also referred to as uniformity or Angular Second Moment (ASM). It measures the uniformity of the textures, i.e. pixel pair replications. It recognizes textural differences $[11,3]$. This measure can be defined according to this formula:

$$
\text { Energ }=\sum_{i, j=0}^{N g} \operatorname{Co}(i, j)^{2}
$$

\subsubsection{Contrast Measure}

This statistic estimates an image spatial frequency. It is the difference between a contiguous set of pixels' highest and lowest values. It measures the amount of local variations present in the image [10]. The equation of contrast is defined as follows:

$$
\text { Contr }=\sum_{i, j=0}^{N g}(i-j)^{2} \operatorname{Co}(i, j)
$$

\subsubsection{Variance Measure}

It's a heterogeneity measure. When the gray levels differ from their mean, the difference increases. The following is the represented formula [11]:

$\operatorname{Var}=\sum_{\mathbf{i}}^{\mathbf{N}}|\mathbf{i}-\mu|^{2} \operatorname{Co}(\mathbf{i}, \mathbf{j})$ 
Where the mean of $\operatorname{Co}(i, j)$ is $\mu$, concentration as

$\mu_{x}$ and $\mu_{y}$

$\mu_{\mathrm{x}}=\sum_{\mathrm{i}} \mathbf{i} \sum_{\mathbf{j}} \mathbf{C o}(\mathbf{i}, \mathbf{j})$

$\mu_{\mathbf{y}}=\sum_{\mathbf{j}} \mathbf{j} \sum_{\mathbf{i}} \operatorname{Co}(\mathbf{i}, \mathbf{j})$

Where $(\mathrm{N})$ is the numbers of pixels

\subsubsection{Auto Correlation Measure}

This measure can be represented by this formula [13]:

Autoc $=\sum_{\mathbf{i}, \mathbf{j}=\mathbf{1}}^{\mathrm{Ng}} \mathrm{ij} \mathrm{Co}(\mathbf{i}, \mathbf{j})$

\subsubsection{IDM (Inverse Difference Moment)}

It is the local homogeneity. This measure can be represented by this formula:

$$
\operatorname{IDM}=\frac{\sum_{i=0}^{N-1} \sum_{j=0}^{N-1} C O(i, j)}{1+(i-j)^{2}}
$$

Where (G) is the gray levels quantized number.

4.3.8. Mean

It is the rate value, where the common brightness of the image and can be determine as : $[12,14]$ :

$$
\breve{g}=\sum_{\mathbf{g}=0}^{\mathrm{N}-1} \operatorname{gp}(\mathrm{g})
$$

Where $(\breve{\mathrm{g}})$ the mean

$\mathrm{P}(\mathrm{g})$ is histogram probability and known as follows:

$$
\mathbf{P}(\mathbf{g})=\frac{N(g)}{M}
$$

Where:

$\mathrm{P}(\mathrm{g})=$ image gray level of g probability .

$\mathrm{N}(\mathrm{g})=$ image number of pixels with gray level $\mathrm{g}$.

$\mathrm{M}=$ image total number of pixel.

\subsubsection{The standard deviation}

It is identified as the square-root of the variance, where the disparity, standard deviation equation is known as [14]:

$\sigma_{g}=\sqrt{\sum_{g=0}^{N-1}(g-\check{g})^{2} P(g)}$

Where $(\mathrm{N})$ is the numbers of pixels 


\subsubsection{Homogeneity Measure}

This statistic is also referred to as IDM. It measures the homogeneity of the image as it proposes greater values for smaller variations in gray tones in elements pair $[15,16]$. The homogeneity equation is defined bellow :

$$
\text { Homg }=\sum_{i, j=0}^{N g} \frac{1}{1+(i-j)^{2}} \operatorname{CO}(i, j)
$$

\subsubsection{Kurtosis}

Qualitatively, kurtosis is a metric to non -specifically describe the peakendness and/or heavy tail shape in a probability distribution through the standardized fourth moment [17].

$$
K=\frac{\left(x^{4}\right)}{\left(x^{2}\right)^{2}}-3
$$

The remaining features are MATLAB instructions.

\subsection{Support vector machine (svm) classification}

An SVM classifier is applied in the proposed work, due to its outstanding, generalization, capability and reputation in the training data set to accomplish high accuracy. Classification of normal and abnormal tumor cases are done using Kernel SVM classifier. An error rate is possible with all classification results and have the probability of either fail to recognize an abnormality, or recognize not existing abnormality. This error rate is defined according to the following terms true and false positive and true and false negative:

True Positive (TP): It is positive for the a clinical abnormality of the classification.

True Negative (TN): It is negative where there are no clinical abnormalities in the classification.

False positive (FP):it is positive for the absence of clinical abnormality in the classification.

False Negative (FN): When the classification leads to clinical abnormality it is negative. Depending on the above parameters three evaluation measures can be concluded:

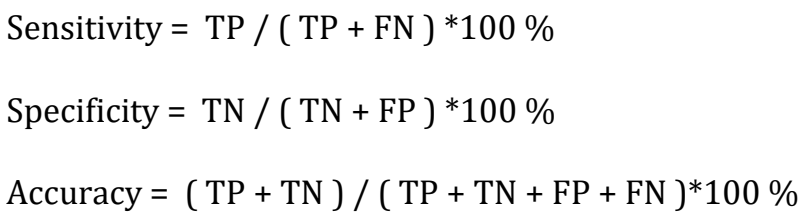

\subsection{Experimental results}

In this work consider total of 20 images contain both benign and malignant Brain tumor MRI, out of these 20 images $30 \%$ of images are used for testing and remaining70\% are used for training. Thirteen features are estimated for each image and then fed to the SVM classifier for training and testing. The 13 extracted features from MRI images for 3 persons as an examples are Illustrated in table (I). 
Table 1 MRI extracted features for three persons.

\begin{tabular}{|l|l|l|l|l|}
\hline Feature number & Features & Image 1 & Image 2 & Image 3 \\
\hline 1 & mean & 0.0031107 & 0.0235179 & 0.00300147 \\
\hline 2 & standard deviation & 0.0897608 & 0.0897839 & 0.0897645 \\
\hline 3 & Entropy & 3.17346 & 3.26983 & 3.55498 \\
\hline 4 & RMS & 0.0898027 & 0.0898027 & 0.0898027 \\
\hline 5 & variance & 0.00804787 & 0.00805116 & 0.00801879 \\
\hline 6 & smoothness & 0.920457 & 0.897422 & 0.9178 \\
\hline 7 & kurtosis & 7.32819 & 7.95668 & 6.36606 \\
\hline 8 & skewness & 0.469022 & 0.886238 & 0.649885 \\
\hline 9 & IDM & -0.0576898 & 0.492585 & 0.472915 \\
\hline 10 & Contrast & 0.208843 & 0.271691 & 0.241379 \\
\hline 11 & correlation & 0.199005 & 0.0930892 & 0.106492 \\
\hline 12 & energy & 0.7621 & 0.76857 & 0.744025 \\
\hline 13 & Homogeneity & 0.93515 & 0.933815 & 0.927651 \\
\hline
\end{tabular}

Table 2 Performance measures of the KSVM method to benign tumor

\begin{tabular}{|l|l|l|l|l|l|l|}
\hline Image & $\begin{array}{l}\text { Sensitivity } \\
\text { \% }\end{array}$ & Specificity\% & $\begin{array}{l}\text { RBF } \\
\text { accuracy }\end{array}$ & $\begin{array}{l}\text { Quadratic } \\
\text { accuracy }\end{array}$ & $\begin{array}{l}\text { Polynomia } \\
\text { laccuracy }\end{array}$ & $\begin{array}{l}\text { Linear } \\
\text { accuracy }\end{array}$ \\
\hline 1 & 87.8094 & 90.4219 & 80 & 80 & 80 & 70 \\
\hline 2 & 68.2927 & 71.8321 & 70 & 80 & 80 & 80 \\
\hline 3 & 82.9227 & 85.6742 & 70 & 90 & 90 & 80 \\
\hline 4 & 92.6829 & 95.3516 & 80 & 90 & 80 & 80 \\
\hline 5 & 90.2439 & 93.6894 & 80 & 80 & 70 & 80 \\
\hline 6 & 87.8049 & 90.3321 & 80 & 90 & 70 & 80 \\
\hline 7 & 82.9268 & 85.2563 & 70 & 90 & 90 & 70 \\
\hline 8 & 75.6098 & 78.6417 & 70 & 90 & 90 & 70 \\
\hline 9 & 85.3656 & 88.7321 & 70 & 90 & 80 & 80 \\
\hline 10 & 90.2439 & 93.3688 & 80 & 90 & 70 & 70 \\
\hline Average & & & 75 & 87 & 80 & 76 \\
\hline
\end{tabular}


Table 3 Performance measures of the KSVM method to malignant tumor.

\begin{tabular}{|l|l|l|l|l|l|l|}
\hline Image & $\begin{array}{l}\text { Sensitivity } \\
\text { \% }\end{array}$ & Specificity\% & $\begin{array}{l}\text { RBF } \\
\text { accuracy }\end{array}$ & $\begin{array}{l}\text { Quadratic } \\
\text { accuracy }\end{array}$ & $\begin{array}{l}\text { Polynomia } \\
\text { laccuracy }\end{array}$ & $\begin{array}{l}\text { Linear } \\
\text { accuracy }\end{array}$ \\
\hline 1 & 93.6829 & 95.2524 & 80 & 90 & 70 & 80 \\
\hline 2 & 85.3659 & 88.9241 & 90 & 80 & 80 & 80 \\
\hline 3 & 97.5612 & 99.5241 & 70 & 90 & 80 & 80 \\
\hline 4 & 95.1225 & 98.7856 & 80 & 90 & 70 & 70 \\
\hline 5 & 97.5632 & 99.6241 & 80 & 80 & 80 & 70 \\
\hline 6 & 92.6829 & 95.3521 & 70 & 90 & 80 & 80 \\
\hline 7 & 95.1224 & 98.2213 & 70 & 80 & 90 & 70 \\
\hline 8 & 80.4878 & 83.7321 & 70 & 80 & 80 & 80 \\
\hline 9 & 93.6132 & 96.8215 & 70 & 80 & 70 & 70 \\
\hline 10 & 88.5672 & 91.3341 & 90 & 100 & 80 & 70 \\
\hline Total & & & 77 & 86 & 78 & 75 \\
\hline
\end{tabular}

According to the above tables the quadratic kernel has the higher accuracy value for both benign (87\%) and malignant $(86 \%)$ tumor MRI images.

Figure 6 shows the SVM accuracy using four different kernels applied to benign and malignant tumor MRI,

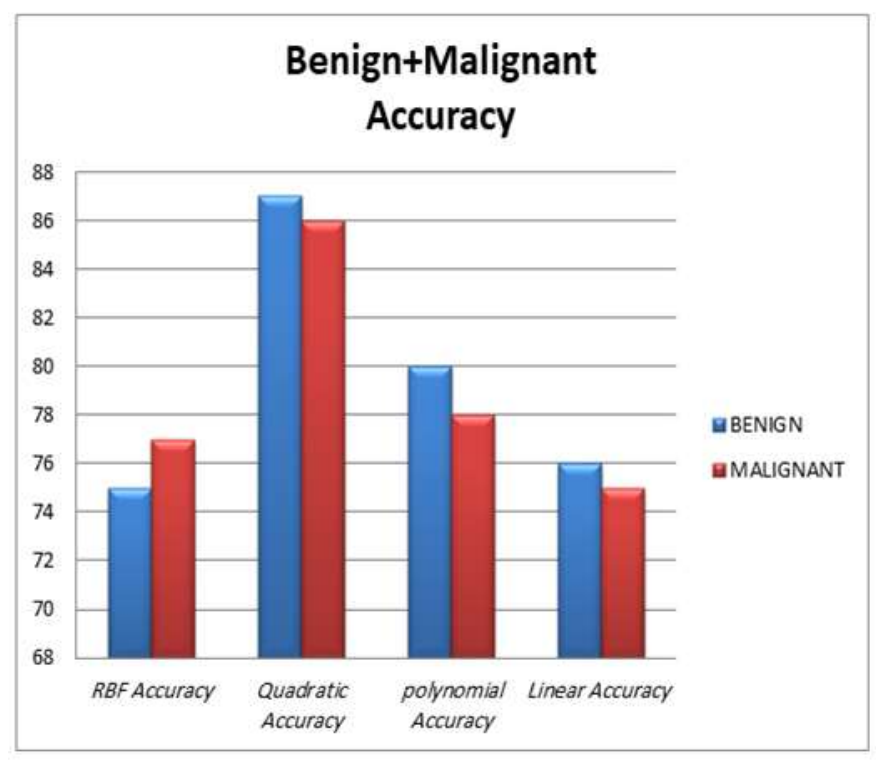

Figure 6 SVM accuracy using four kernels applied to two tumor types

\section{Conclusion}

Identifications brain tumor through MRI images is a hard mission due to the brain complexity. Ten images are selected for benign tumor and ten images for malignant tumor. These images are preprocessed and segmented to allocate the tumor region. Thirteen features were estimated for each image and fed to SVM classifier. For the classification of MRI brain tumor image, a comparison study on the performance of different SVM's kernels is presented. The experiments evaluations shown that the average precision of the SVM classifiers based on a quadratic kernel for 20 images is $86.5 \%$. 
which is higher than other kernels. In future we are trying to increase the dataset of more patients of different age groups.

\section{Compliance with ethical standards}

\section{Acknowledgments}

The authors would like to thank the Mustansiriyah University (www.uomustansiriyah.edu.iq) Baghdad ,Iraq for supporting this work .

\section{Disclosure of conflict of interest}

All authors declare that they have no conflict of interest.

\section{References}

[1] P. Rambabu, C. Naga Raju. "Generation of Random Fields for Object Recognition using Banalization Technique", International Journal of Emerging Trends \& technology in Computer Science (IJETTCS). ISSN 2278-6856. September-October 2015; Volume. 04, Issue: 5(2).

[2] E.A Zanaty, "Determination of gray matter (GM) and white matter (WM) volume in brain magnetic resonance images (MRI)", International Journal of Computer Applications, 2012.

[3] S. Damodharan, D.Raghavan,"Combining tissue segmentation and neural network for brain tumor detection", International Arab Journal of Information Technology, 2015; pp.42-52, vol. 12, no.1.

[4] W.Cui, Y.Wang, Y.Fan, Y.Feng, T.Lei, "Localized FCM clustering with spatial information for medical image segmentation and bias field estimation", International Journal of Biomedical Imaging , 2013; vol. 8 pages , Article ID 930301.

[5] A . Demirhan, I. Guler, M. Toru, "Segmentation of tumor and edema along with healthy tissues of brain using wavelets and neural network", IEEE Journal of Biomedical and Health Informatics,2015; pp. 1451-1458, vol. 19, no. 4.

[6] N. Vani, A.Sowmya, N. Jayamma,"Brain Tumor Classification using Support Vector Machine" , International Research Journal of Engineering and Technology (IRJET), July 2017; Vol. 04.

[7] Y. Zhang, L. Wu, "AN MR BRAIN IMAGES CLASSIFIER VIA PRINCIPAL COMPONENT ANALYSIS AND KERNEL SUPPORT VECTOR MACHINE", School of Information Science and Engineering, Southeast University, Nanjing, China, Progress In Electromagnetics Research, 2012; Vol. 130, 369-388.

[8] Khushbu Isha Vats,. International Journal of Innovative Research in Computer and Communication Engineering. June 2017; Vol. 5, Issue 6.

[9] Amruta B. Patil, J. A . Shaikh, “OTSU Thresholding Method for Flower Image Segmentation",International Journal of Computational Engineering Research (IJCER),2016.

[10] D.A Clausi, "An Analysis of Co-Occurrence Texture Statistics as A Function of Grey Level Quantization", Canadian Journal of remote sensing, 2002; Vol. 28, No. 1, pp. 45-62.

[11] D. Gadkari, "Image Quality Analysis Using GLCM", thesis submitted to the College of Arts and Sciences / University of Central Florida, 2004.

[12] A. Abdullah, S. Jasim, "New Iris Feature Extraction and Pattern Matching Based on Statistical Measurement", International Journal of Emerging Trends \& Technology in Computer Science (IJETTCS), 2014; Vol. 3, No. 5.

[13] L. Soh, C .Tsatsoulis, "Texture Analysis of SAR Sea Ice Imagery Using Gray Level Co-Occurrence Matrices", IEEE Transactions On Geoscience And Remote Sensing, Mar 1999; Vol. 37, No. 2.

[14] K. Abdul-Amir. "Data Acquisition of Digital Images Using Statistical Analysis", Ph.D Thesis, University of Technology, Baghdad, Iraq, 2006.

[15] D .Gadkari, "Image Quality Analysis Using GLCM", thesis submitted to the College of Arts and Sciences/ University of Central Florida, 2004. 
Global Journal of Engineering and Technology Advances, 2021, 07(02), 026-036

[16] L .Soh, C. Tsatsoulis, “Texture Analysis of SAR Sea Ice Imagery Using Gray Level Co-Occurrence Matrices”, IEEE Transactions On Geoscience And Remote Sensing, Mar 1999; Vol. 37, No. 2.

[17] C. Cortes, V. Vapnik, "Support-Vector Networks", Machine Learning, 1995; vol. 20, no. 3, pp. 273-297. 